\title{
Antimicrobial Efficacy of $1 \%$ Chitosan Gel against Periodontal Pathogens: An In Vitro Study
}

\author{
Sreeja Sarkar ${ }^{1}$, Kangowkar V Vandana ${ }^{2}$, Shobha Prakash ${ }^{3}$
}

\begin{abstract}
Aim and objective: The purpose of this study was to evaluate the effects of $1 \%(\mathrm{w} / \mathrm{v})$ chitosan gel as an active agent, on periodontopathogens, Porphyromonas gingivalis, Prevotella intermedia, Fusobacterium nucleatum, and Tannerella forsythia.

Materials and methods: One percent chitosan gel was tested for its antimicrobial activity against four periodontopathogens, P. gingivalis, P. intermedia, F. nucleatum, and T. forsythia by using a disk diffusion test.

Results: The effectiveness of chitosan gel can be measured using its zone of inhibition. Chitosan gel showed the maximum zone of inhibition against $F$. nucleatum. It also produced a wide ring of no bacterial growth where $P$. gingivalis, $P$. intermedia, and $T$. forsythia were inoculated.

Conclusion: In this study, it has been demonstrated that chitosan gel is effective against the periopathogens tested, thereby highlighting its potential to be used in the treatment of chronic periodontitis.

Clinical significance: Chitosan gel shows promise in vitro, by producing a wide zone of no bacterial growth. Therefore, it can be used in the treatment of chronic periodontitis, clinically.

Keywords: Chitosan gel, Disk diffusion test, Periodontopathogens.

CODS Journal of Dentistry (2020): 10.5005/jp-journals-10063-0060
\end{abstract}

\section{INTRODUCTION}

According to the World Health Organization (WHO), 10-15\% of the global population suffer from severe periodontitis. Chronic periodontitis is an infectious disease resulting in inflammation within the supporting tissues of the teeth leading to progressive attachment loss and bone loss. ${ }^{1}$ The inflammatory periodontal diseases are widely accepted as being caused by bacteria associated with dental plaque biofilm. ${ }^{2}$

This accumulation of bacterial biofilm is the main etiological factor of periodontal diseases, which affects the surrounding tissues of the teeth, which may further lead to the destruction of the periodontal attachment apparatus if not treated properly. ${ }^{3}$

Usually, the host response can contain subgingival bacterial challenges, and subclinical infections are resolved without any clinical manifestation of pathosis. However, if the host-parasite equilibrium becomes unbalanced, an exuberant host response can destroy the periodontium. ${ }^{4}$

Both non-surgical and surgical therapy are applicable in the treatment of periodontal disease.

Various pharmacological agents and herbal plants have been tested and employed in the treatment of chronic periodontitis. While they all have shown promising results in their effectiveness against the periodontal pathogens, ${ }^{4}$ the discovery of a novel natural polymer, chitosan has also shown promising potential in all fields including dentistry.

Discovered in 1811 by Henri Braconnot, ${ }^{5}$ chitosan, sometimes known as deacetylated chitin, is a natural polycationic linear polysaccharide derived from partial deacetylation of chitin. ${ }^{6}$ As chitin occurs naturally in fungal cell walls and crustacean shells, it is a fully biodegradable and biocompatible natural polymer, and can be used as an adhesive and as an antibacterial and antifungal agent. ${ }^{7}$

Due to its ability to form gels and films with controlled permeability and good mechanical properties, chitosan has been

\footnotetext{
${ }^{1-3}$ Department of Periodontology, College of Dental Sciences, Davangere, Karnataka, India
}

Corresponding Author: Sreeja Sarkar, Department of Periodontology, College of Dental Sciences, Davangere, Karnataka, India, Phone: +91 8850341589, e-mail: sreejasarkar20@gmail.com

How to cite this article: Sarkar S, Vandana KV, Prakash S. Antimicrobial Efficacy of 1\% Chitosan Gel against Periodontal Pathogens: An In Vitro Study. CODS J Dent 2020;12(2):31-34.

Source of support: For Disk Diffusion Test - Maratha Mandal Research Laboratory and Chitosan Gel Preparation: KLE College of Pharmacy, Hubballi

Conflict of interest: None

considered as a material with potential applications in several fields, such as, agriculture, food, medicine, textiles, and water treatment. Chitosan is a versatile copolymer formed by 2-acetamido-2-deoxyD-glucose and 2-amino-2-deoxy-D-glucose (D-glucosamine) units linked by $O$-glycosidics $\beta$ (1-4) bonds. ${ }^{8}$

It has a broad antimicrobial spectrum against gram-negative, gram-positive bacteria, and fungi. ${ }^{9}$ It also accelerates the formation of osteoblasts which are responsible for bone formation attributing to its regenerative potential on periodontium. ${ }^{10}$

The antimicrobial activity of chitosan formulations was evaluated in a gel/film form against a periodontal pathogen, Porphyromonas gingivalis. The results from this study showed that chitosan could be used as a delivery system for local therapy of periodontal diseases due to its antimicrobial activity and bioadhesive property. ${ }^{11}$

Chitosan and its derivatives have excellent biocompatibility, non-toxicity to human beings, biodegradability, selective permeability, antimicrobial activity, anti-inflammatory, and wound healing properties. One of the most important properties of chitosan is high bioactivity, which makes this material very interesting to develop new biomaterials for application in dentistry. ${ }^{10}$ 
Thus, the study was conducted to evaluate the antimicrobial efficacy of $1 \%$ chitosan gel against the periodontal pathogens, P. gingivalis, Prevotella intermedia, Fusobacterium nucleatum, and Tannerella forsythia. The results of the present study would further be used in the treatment of chronic periodontitis clinically.

\section{Materials and Methods}

For this study, carboxymethyl chitosan was used for the preparation of the gel. The concentration of chitosan gel used in the study was $1 \%(\mathrm{w} / \mathrm{v})$ as it has shown appropriate rheological properties from the applicability point of view. The flow property of the gel at this concentration was found to be suitable for topical application on the oral mucosa and syringe into the periodontal pocket. ${ }^{12}$

\section{Preparation of Chitosan Gel ( $1 \%$ w/v)}

Carboxymethyl chitosan was procured from Everest Biotech Laboratories, Bengaluru. The formulation and evaluation of chitosan hydrogel was carried out at KLE College of Pharmacy, Hubballi, Karnataka. The following steps were carried out:

\section{Method of Preparation}

One gram of carboxymethyl chitosan was weighed accurately and transferred into a dry beaker, to which $50 \mathrm{~mL}$ of distilled water was added and sonicated (Instrument used: Bath sonicator of $20 \mathrm{kHz}$ of frequency) for 15 minutes at room temperature. A clear dispersion of carboxymethyl chitosan hydrogel was formed and the volume was made up to $100 \mathrm{~mL}$ with distilled water. The prepared gel dispersion was evaluated for its appearance, odor, and viscosity and subjected to terminal sterilization before it was sent for disk diffusion test.

\section{Evaluation}

Gel was evaluated for its

\section{Characteristics of the chitosan gel}

\begin{tabular}{ll}
\hline Appearance & Clear light-yellow colored dispersion \\
\hline Odor & Odorless \\
Viscosity & $\begin{array}{l}\text { Evaluated using Brookfield Viscometer D-II } \\
\text { model) L spindle) } 630 \text { centipoise at } 30 \mathrm{rpm}\end{array}$ \\
$\mathrm{pH}$ of the hydrogel & $\begin{array}{l}\text { Measured using Eutech pH meter previously } \\
\text { calibrated to } \mathrm{pH} 4 \text { and } 7 \text { and the } \mathrm{pH} \text { was } \\
\text { found to be } 6.2\end{array}$ \\
\hline
\end{tabular}

\section{Terminal Sterilization}

The prepared hydrogel formulation was filled into clean and dried amber-colored ampoules, sealed, and sterilized at a temperature of $121^{\circ} \mathrm{C}$ for at least 30 minutes by using saturated steam under 15 psi of pressure.

One percent $(w / v)$ chitosan gel was then tested for its antimicrobial activity against four periodontal pathogens; $P$. gingivalis, $P$. intermedia, T. forsythia, and F. nucleatum using disk diffusion test.

\section{Disk Diffusion Test ${ }^{13}$}

The disk diffusion test of the prepared gel was carried out once at Maratha Mandal's Central Research Laboratory.

- Media used:

- Brain heart infusion agar.

- Temperature:

- Agar plates were brought to room temperature before use.
- Inoculum preparation

- Using a loop or swab, the colonies were transferred to the plates.

- Turbidity was adjusted with broth to equal that of a 0.5 McFarland turbidity standard that had been vortexed.

- Inoculation of agar plate

- Within 15 minutes of adjusting the inoculum to a McFarland 0.5 turbidity standard, a cotton sterile swab was dipped into the inoculum to remove excess inoculum.

- The entire surface of the agar plate was swabbed three times, rotating the plates approximately $60^{\circ}$ between streaking to ensure even distribution.

- The inoculated plates were allowed to stand for at least 3 minutes before making wells.

- With the help of hollow tubes, wells were made onto the inoculated agar plates. With the help of a micropipette, chitosan solution was placed on inoculated plates.

- The plates were inverted and incubated with 15 minutes of compound application for 18 to 24 hours at $37^{\circ} \mathrm{C}$ in an incubator.

- The diameter of the inhibition zone was measured to the nearest whole millimeter by holding a measuring device.

\section{Results}

The antimicrobial activity of chitosan was determined by a disk diffusion test. Table 1 presents the inhibition zone diameters determined by disk diffusion of each gram-negative periodontal pathogen. The more sensitive the bacteria is to the chitosan gel, the larger is the clear bacteria-free zone that forms around the chitosan gel.

Of all the periodontal pathogens, F. nucleatum (Fig. 1) showed the maximum zone of inhibition of $18 \mathrm{~mm}$.

The chitosan hydrogel also showed a considerable halo of 12,11 , and $15 \mathrm{~mm}$ revealing the efficiency of the chitosan as an

\begin{tabular}{lll}
\multicolumn{2}{l}{ Table 1: Disk diffusion test } \\
\hline S. no. & Name of the organisms & Chitosan gel \\
\hline 1. & Fusobacterium nucleatum & $18 \mathrm{~mm}$ \\
2. & Porphyromonas gingivalis & $12 \mathrm{~mm}$ \\
3. & Prevotella intermedia & $11 \mathrm{~mm}$ \\
4. & Tannerella forsythia & $15 \mathrm{~mm}$ \\
\hline
\end{tabular}

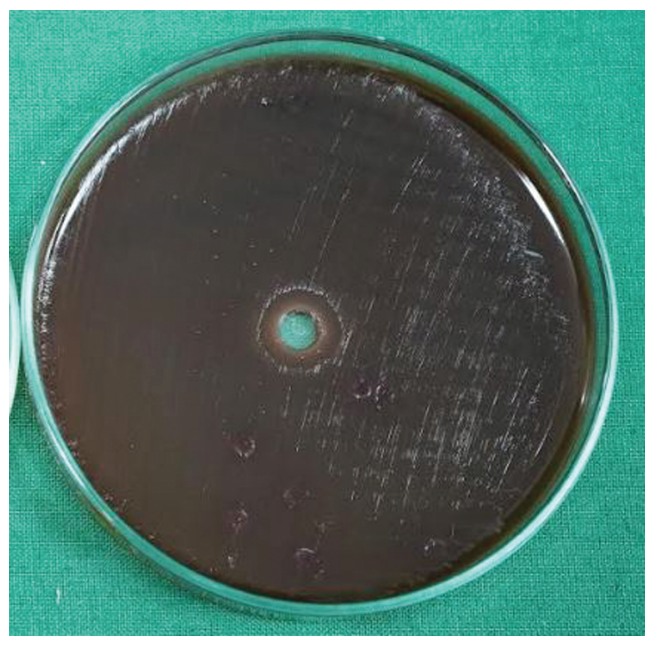

Fig. 1: Zone of inhibition against F. nucleatum 


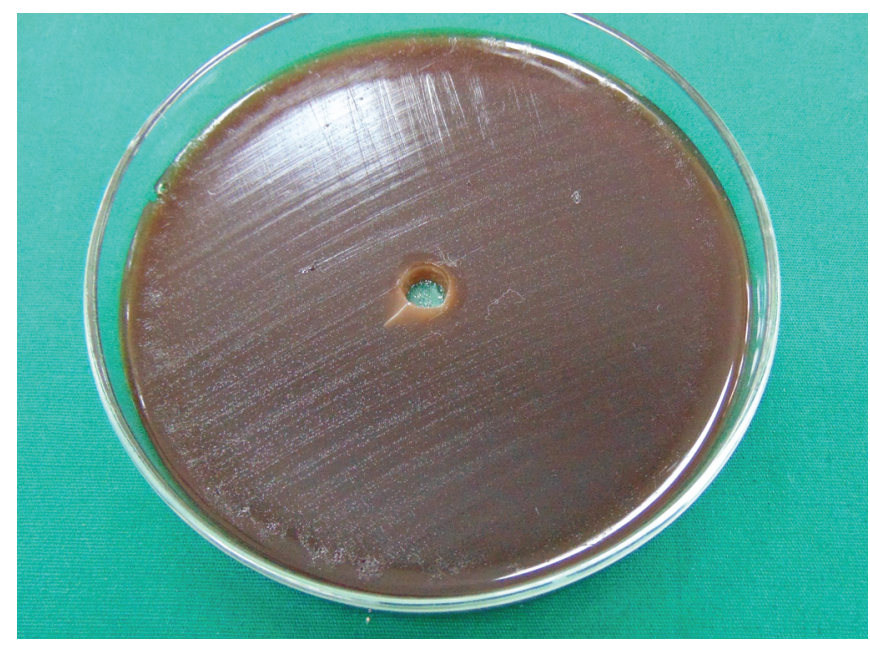

Fig. 2: Zone of inhibition against $P$. gingivalis

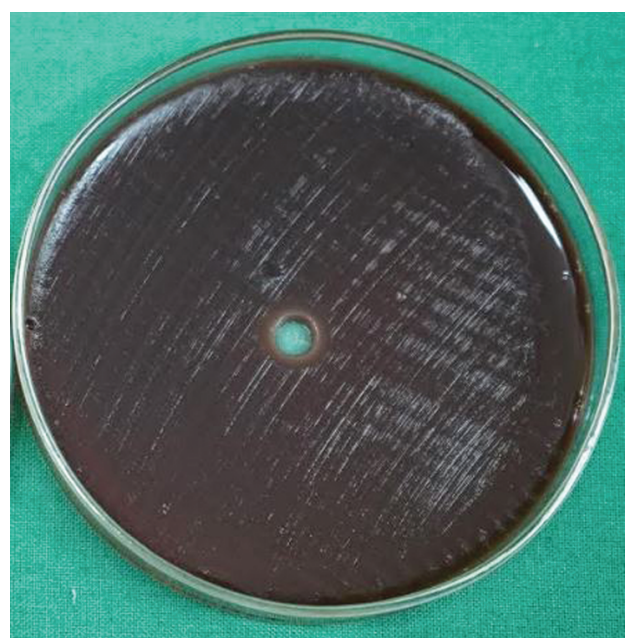

Fig. 4: Zone of inhibition against T. forsythia

antimicrobial agent for P. gingivalis (Fig. 2), P. intermedia (Fig. 3), and T. forsythia (Fig. 4), respectively.

\section{Discussion}

After cellulose, chitin is the second most abundant polysaccharide found in nature. Amidst the innumerable polymers, the alluring properties of this polysaccharide in comparison to other materials of natural origin are that it fails to transmit the potential risk of pathogens of animal origin and does not induce an immune response. $^{14}$

In dentistry, various applications of chitosan have been proposed in which mostly the physical and mechanical properties of this material have been utilized. The commendatory properties of chitosan, such as biodegradability, biocompatibility, nontoxicity, anti-inflammatory, anti-bacterial action, and its ability to be sterilized make it an ideal material in the management of nonsurgical periodontal treatment. ${ }^{14}$

A possible mechanism of antimicrobial action of chitosan is that it possesses reactive positively charged amino groups that interact with the negatively charged bacterial cell membranes, resulting in the leakage of intracellular constituents and alteration of cell permeability, which leads to the death of the microorganism. ${ }^{15}$

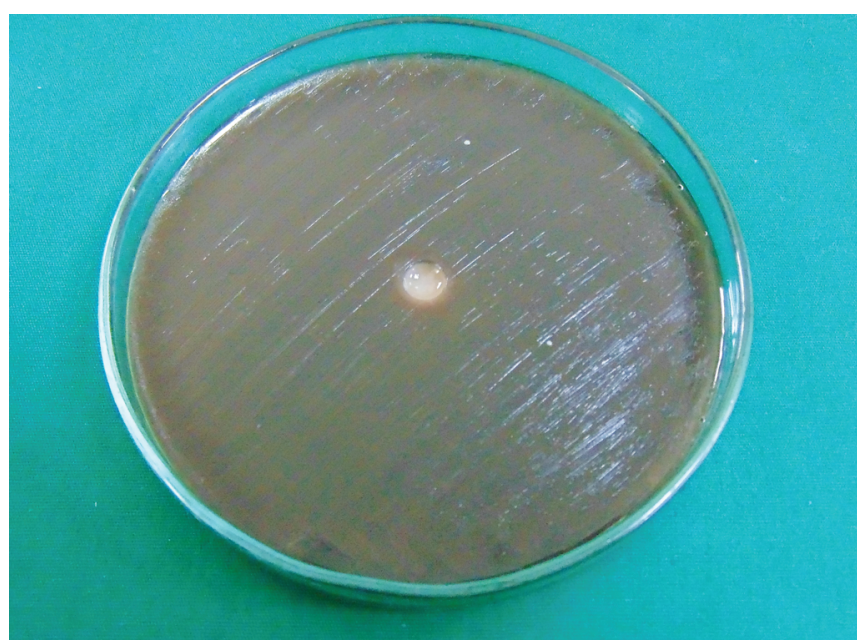

Fig. 3: Zone of inhibition against $P$. intermedia

Numerous studies have been conducted in the past evaluating the antimicrobial efficacy of chitosan and its derivatives. Jeon et al. (2001) and No et al. (2002) reported that chitosan at a concentration of $0.1 \%(\mathrm{w} / \mathrm{v})$ had stronger antimicrobial effects against grampositive bacteria than gram-negative, ${ }^{9}$ although conflicting results were reported in other studies. ${ }^{11}$

For this study, carboxymethyl chitosan was used for the preparation of the gel. Carboxymethyl chitosan is synthesized by introducing a carboxymethyl group in the parent structure of chitosan. It is prepared by carboxymethylation of the hydroxyl and amine moieties of chitosan. This modification increases chitosan's solubility in neutral and basic solutions without affecting any other important characteristics. ${ }^{16}$ The concentration of chitosan gel used in the study was $1 \%(\mathrm{w} / \mathrm{v})$ as it has shown appropriate rheological properties suitable for topical application on the oral mucosa and syringe into the periodontal pocket. ${ }^{12}$

The Kirby-Bauer test for antibiotic susceptibility (also called the disk diffusion test) is a standard test that has been used for years. First developed in the 1950s, it was then standardized by the WHO in 1961. This test is used to determine the resistance or sensitivity of aerobes or facultative anaerobes to specific chemicals, which can then be used by the clinician for the treatment of patients with bacterial infections. The presence or absence of an inhibitory area around the disk identifies the bacterial sensitivity to the drug. ${ }^{17}$

Disk diffusion method is attractive because of its ease of performance and low cost; however, the inhibition zone size is largely determined by how well the compounds uniformly diffuse into the agar media, which may be problematic for many natural compounds that are large and insoluble in water. ${ }^{18}$

Fontana et al. in 2008 conducted a study to evaluate the chitosan gel for its potential application in a controlled drug delivery system. They studied its antimicrobial action using the disk diffusion method in Staphylococcus aureus, an opportunist pathogen found in the microbiota of mucous membrane (buccal, nasal, and oral) and human skin, capable of causing serious infection when penetrated the human organism. Inhibition zone $(11 \mathrm{~mm})$ around chitosan gel was measured after 24 and 48 hours of incubation. ${ }^{19}$

Periodontitis is a chronic inflammatory, polymicrobial disease that leads to the destruction of the supporting tissues of teeth and if left untreated, leads to tooth loss. Periodontal pockets are populated by various periodontal pathogens. The red complex, 
consisting of T. forsythia, P. gingivalis, and Treponema denticola, is strongly associated with severe chronic periodontitis. The orange complex, which includes P. intermedia, F. nucleatum, Campylobacter rectus, and Peptostreptococcus micros, is closely associated with the red complex. The green complex includes Aggregatibacter actinomycetemcomitans, which has a strong association with aggressive periodontitis and a less frequent association with chronic periodontitis. $^{20}$

Non-surgical therapy is the first line of treatment if chronic periodontitis. However, these pathogens may not be accessible to mechanical debridement in areas such as deep periodontal pockets or furcation areas. Using chitosan as a local drug delivery agent adjunct to mechanical debridement can reduce or eliminate the periodontal pathogens from the diseased sites. ${ }^{12}$

In the present study, microbiological analysis of $P$. gingivalis, $P$. intermedia, $T$. forsythia, and F. nucleatum was done as they are associated with Indian adult periodontitis and rapidly progressive periodontitis patients.

The results of the disk diffusion test showed the zones of inhibition around the chitosan gel; $12,11,15$, and $18 \mathrm{~mm}$ for $P$. gingivalis, P. intermedia, T. forsythia, and $F$. nucleatum, respectively. These inhibition zone diameters indicate that the periodontal pathogens are susceptible to $1 \%$ chitosan gel. This correlates well with the results of the study where the antimicrobial efficacy of the chitosan was evaluated against $P$. gingivalis. ${ }^{11}$

However, further microbial evaluations and statistical analysis of different microbial tests are needed to attest to the antimicrobial efficacy of the chitosan formulation used in the study.

Within the limitations of the study, the results from the present study suggest a promising application of chitosan in the treatment of chronic periodontitis. These results support the antimicrobial effect of $1 \%$ chitosan gel against the periodontal pathogens and its application in in vivo testing.

\section{Conclusion}

Chitosan is a natural antimicrobial that combines several favorable biological characteristics, including biodegradability, biocompatibility, and non-toxicity. From the results of the study, it can be concluded that with its bioadhesive and antimicrobial properties, chitosan in gel or film form can be a promising delivery for antibacterial drugs in periodontal therapy. However, future studies and microbial evaluations are needed.

\section{Clinical Significance}

In this study, the results showed effective antimicrobial efficacy of $1 \%(w / v)$ chitosan gel against the Gram-negative periodontal pathogens. Within the limitations of this study, chitosan gel can be formulated into gels, films as a vehicle for drug delivery of antimicrobial agents, or can be used as an active agent in itself to extend its use in the treatment of chronic periodontitis. However, extensive in vitro and in vivo tests are needed to conclude its treatment in chronic periodontitis.

\section{References}

1. Cohen R, Mariotti A, Rethman M, et al. Glossary of Periodontal Terms. 4th ed., Chicago: American Academy of Periodontology; 2001. p. 40.

2. Socransky SS, Haffajee AD. The bacterial etiology of destructive periodontal diseases: current concepts. J Periodontol 1992;63(4s):322331. DOI: 10.1902/jop.1992.63.4s.322.

3. da Rocha Júnior HA, Silva C, Santiago F, et al. Local drug delivery systems in the treatment of periodontitis: a literature review. J Int Acad Periodontol 2015;17(3):82-90.

4. Greenstein G. Local drug delivery in the treatment of periodontal diseases: assessing the clinical significance of the results. J Periodontol 2006;77(4):565-578. DOI: 10.1902/jop.2006.050140.

5. Kavitha K, Keerthi TS, Mani TT. Chitosan polymer used as carrier in various pharmaceutical formulations: brief review. Int J Appl Biol Pharmaceut Technol 2011;2(2):249-258.

6. Chandy T, Sharma CP, 1995. Resorbable chitosan matrix-a promising biomaterials for the future. Biomed. Engg. Conf., Proc. of the Fourteenth Southern, pp: 282-285. New York: IEEE.

7. Cheung RC, Ng TB, Wong JH, et al. Chitosan: an update on potential biomedical and pharmaceutical applications. Mar Drugs 2015;13(8):5156-5186. DOI: 10.3390/md13085156.

8. Elieh-Ali-Komi D, Hamblin MR. Chitin and chitosan: production and application of versatile biomedical nanomaterials. Int J Adv Res 2016;4(3):411.

9. Goy RC, Britto DD, Assis OB. A review of the antimicrobial activity of chitosan. Polímeros 2009;19(3):241-247. DOI: 10.1590/S010414282009000300013.

10. Kmiec M, Pighinelli L, Tedesco MF, et al. Chitosan-properties and applications in dentistry. Adv Tissue Eng Regen Med Open Access 2017;2(4):00035.

11. Ikinci G, Senel S, Akincibay H, et al. Effect of chitosan on a periodontal pathogen porphyromonas gingivalis. Int J Pharmaceut 2002;235(12):121-127. DOI: 10.1016/S0378-5173(01)00974-7.

12. Akincibay $H$, Senel $S$, Ay ZY. Application of chitosan gel in the treatment of chronic periodontitis. J Biomed Mat Res Part B: Appl Biomater 2007;80(2):290-296. DOI: 10.1002/jbm.b.30596.

13. Isenberg HD. Clinical microbiology procedures handbook, vol. 1, Washington D.C: American Society for Microbiology; 1992.

14. Babrawala IS, Prabhuji ML, Karthikeyan BV, et al. A novel approach using natural $1 \%(\mathrm{~W} / \mathrm{W})$ chitosan as a local drug delivery system in the management of non-surgical periodontal treatment: a pilot study. $J$ Int Acad Periodontol 2016;18(4):129-133.

15. Raafat D, Sahl HG. Chitosan and its antimicrobial potential-a critical literature survey. Microb Biotechnol 2009;2(2):186-201. DOI: 10.1111/j.1751-7915.2008.00080.x.

16. Asiri AM, Mohammad A. Applications of nanocomposite materials in drug delivery. Woodhead Publishing; 2018.

17. Hudzicki J, Kirby-Bauer disk diffusion susceptibility test protocol. 8th December 2009.

18. Jiang L, Wang F, Han F, et al. Evaluation of diffusion and dilution methods to determine the antimicrobial activity of water-soluble chitosan derivatives. J Appl Microbiol 2013;114(4):956-963. DOI: 10.1111/jam.12111.

19. Fontana CR, dos Santos Junior DS, Bosco JM, et al. Evaluation of chitosan gel as antibiotic and photosensitizer delivery. Drug Deliv 2008;15(7):417-422. DOI: 10.1080/10717540802007433.

20. Slots J, Ting M. Actinobacillus actinomycetemcomitans and Porphyromonas gingivalis in human periodontal disease: occurrence and treatment. Periodontol 2000 1999;20(1):82-121. DOI: 10.1111/ j.1600-0757.1999.tb00159.x. 